\title{
CARACTERÍSTICAS CLÍNICAS Y PRONÓSTICO DE LAS PACIENTES CON CÁNCER DE CÉRVIX TRATADAS EN TRES INSTITUCIONES DE SALUD EN BARRANQUILLA, COLOMBIA, DE 2005 A 2011
}

\section{Clinical characteristics and prognosis of patients with cervical cancer treated in three healthcare centres in Barranquilla, Colombia, between \\ 2005 and 2011}

Róbinson Fernández-Mercado, $M D^{1}$; Jairo Amaya-Guío, $M D^{2}$; Álvaro González-Rubio, $\mathrm{MD}^{3}$; Rafael Pineda-Vega, $\mathrm{MD}^{4}$; Elbert Riveros-Torrado, $\mathrm{MD}^{5}$; Angélica Álvarez-González, MD ${ }^{5}$; José M. Corro-Melgarejo, $M D^{5}$

Recibido: abril 22/13 - Aceptado: junio 24/14

\section{RESUMEN}

Objetivo: describir las características clínicas y el pronóstico de las pacientes con cáncer de cérvix tratadas en tres instituciones de salud en Barranquilla, Colombia.

Materiales y métodos: estudio de cohorte retrospectiva descriptiva. Se revisaron las bases de datos de las tres instituciones participantes en donde se identificaron los casos que tuvieron diagnóstico CIE 10 de: tumor maligno de endocérvix, tumor maligno de exocérvix, tumor maligno del cuello del útero y tumor maligno del itsmo uterino. Se excluyeron

1 Ginecólogo oncólogo. Docente de posgrado, Universidad Libre y Universidad Metropolitana de Barranquilla, Colombia. rosefeme2002@yahoo.es

2 Ginecoobstetra. Epidemiólogo Clínico. Profesor Asociado, Facultad de Medicina, Universidad Nacional de Colombia, Bogotá, Colombia.

3 Ginecólogo-Oncólogo, Docente postgrado Universidad libre y Universidad Metropolitana de Barranquilla, Colombia.

4 Médico Epidemiólogo, Universidad Corpas, Bogotá, Colombia.

5 Residente de ginecoobstetricia, tercer año, Universidad Metropolitana de Barranquilla, Colombia. pacientes remitidas por recidiva tumoral. En caso de deceso, este fue corroborado con el certificado de defunción. Se describe la mortalidad absoluta por estadio clínico. Para establecer el pronóstico se estimó la probabilidad de estar libre de enfermedad y de sobrevivir al primer, tercer y quinto año de seguimiento.

Resultados: se identificaron 375 pacientes con diagnóstico de carcinoma de cérvix, de las cuales 27 se perdieron en el seguimiento para un total de 348 pacientes evaluadas. Se encontró una tasa de complicaciones cercana al 19\% siendo más frecuentes en pacientes con radioterapia (94\%) que en las que recibieron tratamiento quirúrgico (6\%). La probabilidad de sobrevida a los 5 años es del $92 \%$ y la probabilidad de estar libre de enfermedad a los 5 años es del $89 \%$.

Conclusiones: los resultados obtenidos en cuanto a complicaciones y sobrevida posterior al tratamiento fueron similares a lo reportado en la literatura. Se requieren más estudios con una mayor profundidad 
metodológica que caractericen el pronóstico de las pacientes con cáncer de cérvix en nuestra población. Palabras clave: cáncer de cérvix, escamocelular, adenocarcinoma, estadio clínico, pronóstico.

\section{ABSTRACT}

Objective: To describe the clinical characteristics and the prognosis of patients with cervical cancer treated in three healthcare centres in Barranquilla, Colombia.

Materials and methods: Descriptive retrospective cohort study. The databases of the three participating centres where reviewed to identify the cases with a diagnosis of CIE 10: malignant endocervical tumour, malignant exocervical tumour, malignant uterine cervical tumour, and malignant tumours of the uterine isthmus. Patients referred due to tumour relapse were excluded. Death certificates were used to confirm mortality cases. Absolute mortality is described by clinical stage. To establish prognosis, probability of disease-free survival at 1 , 3 and 5 years of following was estimated.

Results: Overall, 375 patients diagnosed with cervical carcinoma were identified. Of them, 27 were lost to follow-up, for a total of 348 patients assessed. A rate of complications of approximately $19 \%$ was found, complications being more frequent among patients receiving radiotherapy (94\%) than among those receiving surgical treatment (6\%). The 5 -year survival probability is $92 \%$ and the probability of being disease-free after five years is $89 \%$.

Conclusions: Results in terms of complications and survival after treatment were similar to those reported in the literature. More studies and with a better methodological quality are required in order to characterize the prognosis for patients with cervical cancer in our population.

Key words: Cervical cancer, squamous cell, adenocarcinoma, clinical stage, prognosis.

\section{INTRODUCCIÓN}

El cáncer de cérvix es el tercero en frecuencia y constituye la cuarta causa de muerte por cáncer en mujeres alrededor del mundo (1). Para el año 2008 se estimó que 530.232 mujeres fueron diagnosticadas con esta entidad ocurrieron 275.008 muertes, siendo África, Asia y América Latina las poblaciones que reportan una mayor tasa de mortalidad. En Estados Unidos, aproximadamente 12.170 mujeres fueron diagnosticadas con cáncer de cérvix y 4.220 murieron a causa de la enfermedad en 2012 (2). En América Latina el cáncer de cérvix es considerado como un problema de salud pública que afecta predominantemente a las mujeres en edad reproductiva y económicamente menos favorecidas. De acuerdo con el Departamento Administrativo Nacional de Estadística (DANE), en el departamento del Atlántico (Colombia), el cáncer de cérvix ocupó durante el año 2010 el segundo lugar en frecuencia (3) con una incidencia estimada de 21,5 casos por 100.000 mujeres, superado solo por el cáncer de seno (con una incidencia de 31,2 casos por cada $100.000 \mathrm{mu}-$ jeres), pero ocupó el primer puesto en mortalidad por cáncer (tasa de mortalidad 10 x 100.000) (4).

Múltiples estudios han permitido identificar la presencia de factores de riesgo asociados al desarrollo de esta neoplasia siendo el inicio temprano de las relaciones sexuales, el antecedente de múltiples compañeros sexuales, la multiparidad (5), el antecedente de infecciones de transmisión sexual, el tabaquismo, la inmunosupresión, la conducta sexual de riesgo, la desnutrición y el uso de anticonceptivos orales los reportados con mayor frecuencia (6).

Se ha establecido que mujeres con dos o más hijos tienen un riesgo $80 \%$ mayor de presentar lesiones intraepiteliales del cérvix, con respecto a las nulíparas (5). Diversos estudios han mostrado que los compañeros sexuales de las mujeres con diagnóstico de cáncer de cérvix tienen con mayor frecuencia el antecedente de un número elevado de compañeras sexuales, sexo comercial, tabaquismo e incluso cáncer de pene $(7,8)$.

El tratamiento del cáncer de cérvix y su pronóstico dependen del estado clínico al momento del diagnóstico e incluye cirugía radical o conservadora en estadios iniciales (IA hasta IB1) o radioterapia 
más quimioterapia concomitante o radioterapia sola en casos más avanzados. Algunos centros realizan también cirugía radical a los pacientes con enfermedad en estadios IIA y IIB inicial (9). Es poco lo que se conoce en nuestra población respecto a las complicaciones de las diferentes alternativas de tratamiento y el pronóstico.

El objetivo del presente estudio es describir las características clínicas, las complicaciones asociadas al tratamiento y el pronóstico de las pacientes con diagnóstico de cáncer de cérvix que fueron tratadas en tres instituciones de salud de la ciudad de Barranquilla.

\section{MATERIALES Y MÉTODOS}

Estudio de cohorte retrospectiva, descriptivo. Se incluyeron pacientes con diagnóstico histopatológico de cáncer de cérvix tratadas en cualquiera de las tres instituciones participantes durante el periodo de estudio, con al menos un año de seguimiento, que ingresaron entre el 1 de enero de 2005 al 31 de diciembre de 2011. El seguimiento se hizo hasta que cumplieron 5 años de tratamiento o hasta el mes de julio de 2012, fecha en que fueron censurados los datos de los pacientes con menos de 5 años de seguimiento. Se excluyeron las pacientes remitidas por recidiva tumoral.

El estudio se llevó a cabo en tres instituciones de alta complejidad en la ciudad de Barranquilla: Clínica Julio Enrique Medrano (Saludcoop EPS) que atiende población perteneciente al régimen contributivo, la Unidad de Ginecología Oncológica Misión Médica y la Clínica Bonnadona Prevenir que atienden pacientes pacientes del régimen de aseguramiento contributivo y del régimen subsidiado por el Estado.

Muestreo y tamaño muestral. Se realizó un muestreo por conveniencia a partir del universo de pacientes atendidas en las instituciones participantes durante el periodo de estudio. Se tomaron en cuenta todas las pacientes con este diagnóstico que acudieron a las tres instituciones antes mencionadas en el periodo descrito.
Procedimiento. Previo diseño de un instrumento de recolección con las variables de interés, los investigadores revisaron la base de datos de las tres instituciones donde se identificaron los casos que tuvieran diagnóstico CIE 10 de: tumor maligno de endocérvix (C530), tumor maligno de exocérvix (C531), tumor maligno del cuello del útero (C539), tumor maligno del istmo uterino (C540). Una vez identificados los casos se revisó la historia clínica de cada una de las pacientes aplicando los criterios de inclusión y de exclusión. Se registraron las variables en el instrumento diseñado para tal fin y se revisó la evolución consignada en la historia clínica. En caso de deceso, este fue corroborado con el certificado de defunción.

Las variables por medir fueron: edad, paridad, edad al inicio de la vida sexual, número de compañeros sexuales, tabaquismo, tipo histológico, estadio clínico, tratamiento recibido y condición final (libre de enfermedad o muerte).

Análisis. Para el análisis de las variables se utilizó el modelo EPI info versión 3.5.1. Se realizó estadística descriptiva para las características clínicas y sociodemográficas al ingreso. Se calculó la media y la desviación estándar o las medianas y el rango según el tipo de variable, y se calcularon proporciones para las variables categóricas. Se describe la mortalidad absoluta por estadio en los 5 años. A fin de establecer el pronóstico se revisó la condición final (probalidad de estar libre de enfermedad o sobrevivir) al primero, segundo, tercero, cuarto y quinto año de seguimiento mediante el cálculo de probabilidad condicional (1 - probabilidad de recurrir dado que no había recurrido la enfermedad en el periodo inmediatamente anterior y 1 - probabilidad de morir dado que no había fallecido en el periodo inmediatamente anterior).

Aspectos éticos. El estudio fue aprobado por el Comité de Ética de la Unidad de Ginecología Oncológica Misión Médica Ltda., Clínica Bonnadona - Prevenir y Clínica Julio Enrique Medrano de SaludCoop. 


\section{RESULTADOS}

Se identificaron 375 pacientes con diagnóstico de carcinoma de cérvix a partir de la búsqueda realizada en la base de datos de las tres instituciones. De estas, en 27 (7,2\%) no se contó con el registro completo de la información o no había información disponible con respecto al seguimiento. No hubo pacientes con remisión por recidiva tumoral. Finalmente, un total de 348 pacientes fueron tomadas en cuenta para este análisis descriptivo.

La edad promedio al momento del diagnóstico fue de 52 años (desviación estándar 13,3), y el rango de edad al inicio de la vida sexual fue de 12 a 30 años con una mediana a los 18. La paridad osciló en un rango de 0 a 18 hijos (mediana: 4), el número de compañeros sexuales tuvo una mediana de 2 (rango de 1 a 10). El $32 \%$ de las pacientes reportaron el uso del cigarrillo. El carcinoma escamocelular se diagnosticó en el 81,3\% de las pacientes y el adenocarcinoma en el 18,7\%.

El estadio clínico IIB fue el más frecuentemente diagnosticado con un 33,6\% (117 pacientes), seguido por el IB1 con un 27,3\% (95 pacientes). En 68 pacientes (19,3\%) no se pudo documentar el estadio clínico debido a que fueron tratadas inicialmente en otras instituciones, pero el seguimiento se hizo en alguna de las tres instituciones del estudio (figura 1).

La radioterapia más quimioterapia concomitante fue el tratamiento realizado en 145 pacientes (41,9\%), seguido por la radioterapia sola en 131 pacientes $(37,6 \%)$ y el tratamiento quirúrgico en 72 pacientes (20,7\%). La técnica más utilizada fue la histerectomía radical más linfadenectomía pélvica (Clase III de Rutledge para los estadios IB1 y IB2) que se realizó en 63 de las apacientes sometidas a cirugía (figura 2).

Se reportaron complicaciones asociadas al tratamiento en 69 pacientes (19,8\%): de ellas, 65 $(18,6 \%)$ recibieron radioterapia sola o asociada a quimioterapia (proctitis en 54 pacientes, fístula rectovaginal en 6 pacientes, fístula vesical y colostomía en 2 pacientes y nefrectomía en una paciente) y 4 (5,6\%) en pacientes sometidas a cirugía (incontinencia fecal y linfedema de miembros inferiores, infección de sitio operatorio e hidronefrosis).

El seguimiento de las pacientes es el siguiente: 348 cumplieron un año de seguimiento; 259, dos años; 177, tres años; 137, cuatro años y 100, cinco años (tabla 1). Se presentaron 32 recaídas al final del seguimiento (31 de julio de 2012), el $75 \%$ la

Figura 1.

Estadios clínicos al momento del diagnóstico del cáncer de cérvix

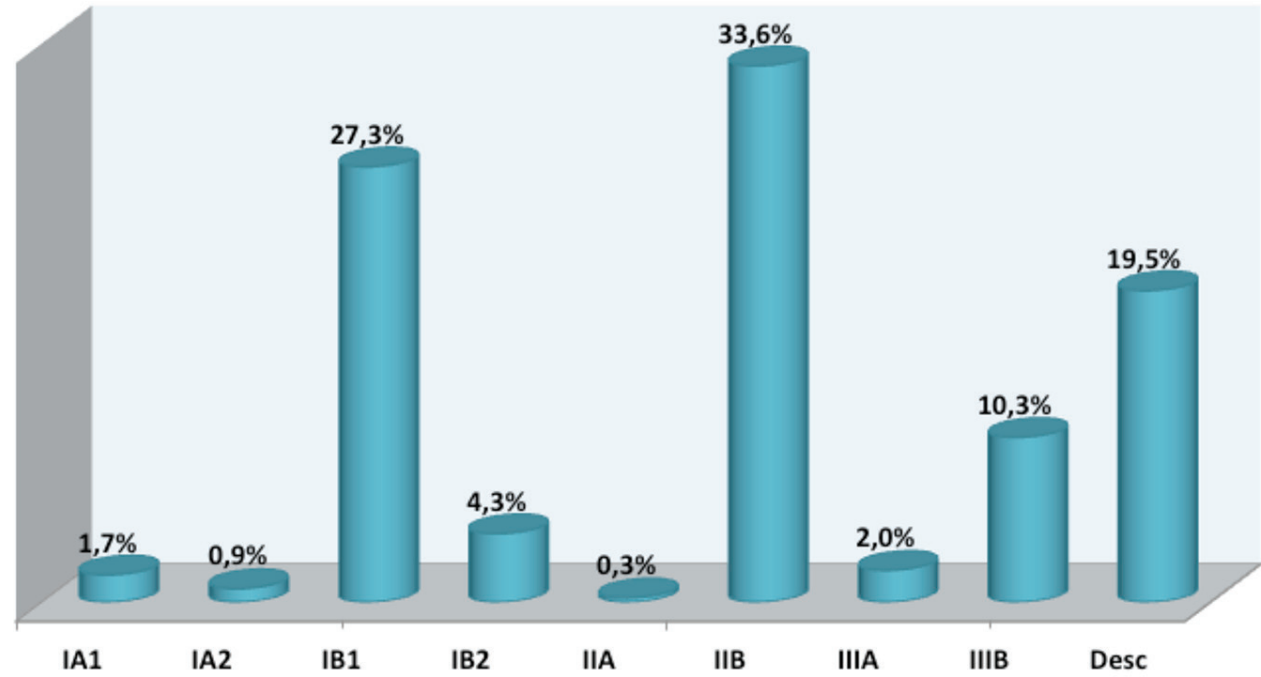

Fuente: historias clínicas Misión Médica, Saludcoop EPS y Clínica Bonnadona Prevenir. 
Figura 2.

Distribución de tratamiento inicial según estadio clínico del cáncer de cérvix

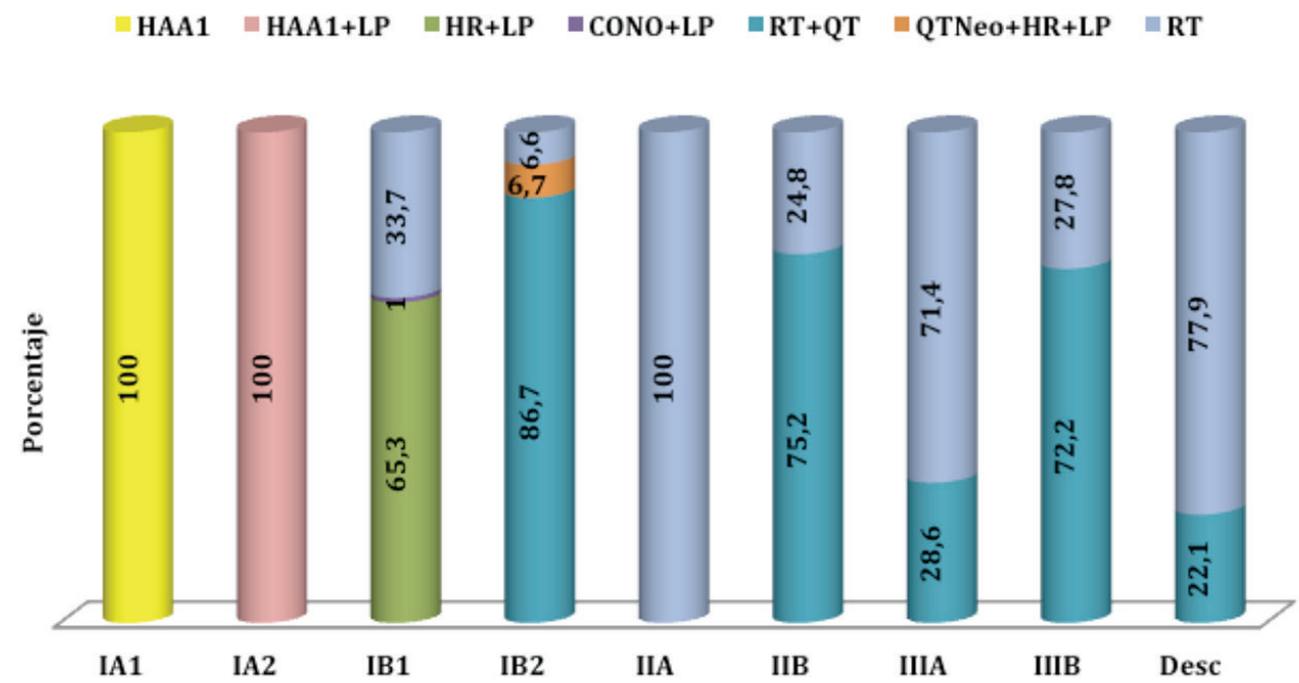

HAA1: histerectomía abdominal ampliada clase 1, LP: linfadenectomía pélvica, HR: histerectomía radical, CONO: conizacion, RT: radioterapia, QT: quimioterapia, QT Neo: quimioterapia neoadyuvante, RT: radioterapia

Fuente: historias clínicas Misión Médica, Saludcoop EPS y Clínica Bonnadona Prevenir.

desarrolló al primer año, el 18,8 \% al término del tercer año y un 6,2\% al final del quinto año (tabla 1). Durante el seguimiento a 5 años la probabilidad de estar libres de enfermedad es del 88,8\% (tabla 2). Con respecto a la mortalidad se presentaron 18 muertes al final del seguimiento (31 de julio de 2012): 4 pacientes con estadio FIGO IB1, 6 pacientes con estadio IIB, 5 con estadio IIIB y 3 con estadio desconocido. El 77,8\% de las muertes se presentaron en los dos primeros años de seguimiento (tabla 1). La probabilidad de sobrevivir 5 años después del tratamiento es del 92,2\% (tabla 2).

\section{DISCUSIÓN}

Este estudio mostró una tasa de complicaciones cercana al 19\%, siendo más frecuentes en pacientes con radioterapia (94\%) que en las que recibieron tratamiento quirúrgico (6\%). La sobrevida es cercana al $90 \%$ a los 5 años.
En nuestro estudio las complicaciones asociadas a la cirugía fueron similares a las reportadas por Marin et al. en pacientes sometidas a histerectomía Clase III de Rutledge (5,6 frente a 5,4\% respectivamente). En su publicación Marin et al. reportan complicaciones como íleo dinámico (2,7\%), hematoma supra-aponeurótico $(5,4 \%)$ y sangrado intraabdominal (2,7\%) que en nuestro estudio no encontramos probablemente debido a que los procedimientos quirúrgicos están estandarizados y fueron realizados únicamente por dos de los investigadores (Robinson Fernández y Álvaro González) (10). El tratamiento con radioterapia más quimioterapia concomitante o radioterapia sola produce efectos secundarios a corto, mediano y largo plazo. Los tejidos que normalmente tienen riesgo de desarrollar complicaciones asociadas a la radioterapia son la vejiga, el rectosigmoide y el intestino delgado. La severidad de las complicaciones 


\begin{tabular}{|c|c|c|c|c|c|c|c|c|c|c|}
\hline \multirow{3}{*}{$\begin{array}{l}\text { Año de } \\
\text { ingreso }\end{array}$} & \multicolumn{10}{|c|}{ Seguimiento a } \\
\hline & \multicolumn{2}{|c|}{$\begin{array}{c}1 \text { año } \\
(\mathrm{N}=348)\end{array}$} & \multicolumn{2}{|c|}{$\begin{array}{c}2 \text { años } \\
(\mathrm{N}=259) \\
\end{array}$} & \multicolumn{2}{|c|}{$\begin{array}{c}3 \text { años } \\
(N=177)\end{array}$} & \multicolumn{2}{|c|}{$\begin{array}{c}4 \text { años } \\
(\mathrm{N}=137) \\
\end{array}$} & \multicolumn{2}{|c|}{$\begin{array}{c}5 \text { años } \\
(N=100)\end{array}$} \\
\hline & Recaída & Mort & Recaída & Mort & Recaída & Mort & Recaída & Mort & Recaída & Mort \\
\hline 2005 & 2 & 0 & 0 & 1 & 2 & 0 & 2 & 1 & 0 & 2 \\
\hline 2006 & 2 & 0 & 0 & 2 & 0 & 0 & 0 & 0 & 0 & 0 \\
\hline 2007 & 0 & 0 & 0 & 0 & 1 & 0 & 0 & 1 & 0 & 0 \\
\hline 2008 & 5 & 1 & 0 & 3 & 0 & 0 & 0 & 0 & & \\
\hline 2009 & 2 & 0 & 0 & 2 & 2 & 0 & & & & \\
\hline 2010 & 8 & 3 & 1 & 1 & & & & & & \\
\hline \multirow[t]{2}{*}{2011} & 5 & 1 & & & & & & & & \\
\hline & Total & 24 & 5 & 1 & 9 & 5 & 0 & 2 & 2 & 02 \\
\hline Porcentaje & $6,9 \%$ & $1,4 \%$ & $0,4 \%$ & $3,5 \%$ & $2,8 \%$ & $0,0 \%$ & $1,5 \%$ & $1,1 \%$ & $0,0 \%$ & $2,0 \%$ \\
\hline
\end{tabular}

Fuente: historias Clínicas Misión Médica, Saludcoop EPS y Clínica Bonnadona Prevenir

Tabla 2.

Probabilidad de estar libre de enfermedad o de sobrevivir en pacientes tratadas por cáncer de cérvix

\begin{tabular}{|l|c|c|c|c|c|c|}
\hline \multirow{2}{*}{} & \multicolumn{5}{|c|}{ Probabilidad } \\
\cline { 2 - 7 } & 1 año (\%) & 2 años (\%) & a 3 años (\%) & a 4 años (\%) & a 5 años (\%) \\
\hline Libre de enfermedad & 93,1 & 92,7 & 90,1 & 88,8 & 88,8 \\
\hline De sobrevivir & 98,6 & 95,1 & 95,1 & 94,1 & 92,2 \\
\hline
\end{tabular}

está relacionada con la dosis. Wang, en una revisión sistemática de la literatura, reportó que la incidencia de complicaciones severas en vejiga, rectosigmoide e intestino delgado fueron: 1,8 frente a $1,3 \% ; 3,1$ frente a $3,0 \%$, y 2,0 frente a $0,5 \%$ para radioterapia de alta y baja tasa respectivamente (11). ZobecLogar et al., en pacientes sometidas a tratamiento con radioterapia más quimioterapia reportaron una toxicidad gastrointestinal del 15,3\% (12). Nuestros resultados con radioterapia de alta tasa son más altos en cuanto a proctitis $(19,6 \%)$ y similares en los otros tejidos (vejiga e intestino delgado); no se determinó la agudeza de dicha sintomatología como es reportada en otros estudios.

En la población estudiada se encontró que un 90,8\% están libres de enfermedad, así como una mortalidad global de 5,5\% a los 5 años, similar a lo publicado en la literatura que muestra una sobrevida de $80-90 \%$ a 5 años para estadios IB y IIA, de 50-80\% para estadio IIB y de 25-50\% para estadio III. Green et al., en un estudio colaborativo, encontraron que la radioterapia más quimioterapia concomitante mejora la sobrevida y el periodo libre de enfermedad entre un 10 y $13 \%$, sea o no 
el cisplatino el fármaco utilizado (13); en nuestro estudio no se exploró esta asociación.

La edad promedio en la cual se diagnostica el cáncer invasivo de cuello uterino generalmente es la cuarta década de la vida; una revisión de doce estudios epidemiológicos publicada por The International Collaboration of Epidemiological Studies of Cervical Cáncer en el año 2006 muestra que la edad promedio de diagnóstico de cáncer escamocelular fue de 45 (rango 37 a 57 años) y de 43 años (rango 32 a 55) para adenocarcinoma, en el presente estudio para los dos tipos de cáncer la edad promedio fue mayor (52 años), probablemente debido a que nuestras usuarias están llegando tarde a los servicios de salud y con estadios avanzados de la enfermedad por ineficiencia de los programas de tamizaje (14).

El carcinoma escamocelular sigue siendo el tipo histológico más frecuente entre las patologías malignas del cérvix, datos que son similares a los del presente estudio (15). La estadificación clínica mostró que la mayoría de nuestras pacientes presentaban un cáncer de cérvix localmente avanzado, siendo el estadio IIB el más frecuente con un 33,6\% de los casos; el uso de esta clasificación es cuestionado por diversos estudios que la consideran poco precisa para definir la extensión de la enfermedad (16).

El tratamiento del cáncer invasivo abarca el adecuado manejo de la lesión primaria y de los focos potenciales de enfermedad metastásica. El enfoque primario incluye cirugía y radioterapia, aunque la primera suele reservarse a pacientes con estadios I o IIA tempranos; algunos centros europeos y asiáticos realizan cirugía primaria a pacientes en estadio IIB inicial (17).

El 20,7\% de las pacientes fueron sometidas a cirugía (histerectomía radical junto con linfadenectomía pélvica bilateral). El manejo conservador (traquelectomía radical con linfadenectomía pélvica bilateral) está reservado para pacientes que deseen fertilidad y con enfermedad estadio IA1 con invasión extensa del espacio linfo-vascular, IA2 y IB1 menor o igual a dos centímetros de diámetro sin afectación del endocérvix superior en la resonancia magnética o en la sección congelada intraoperatoria (18). A partir de la publicación de cinco estudios clínicos controlados en 1999, y siguiendo a las orientaciones del Instituto Nacional de Cáncer de Estados Unidos, se ha producido un cambio significativo en el tratamiento del cáncer cervical avanzado con un aumento del número de paciente que reciben quimiorradiación simultánea: del $20 \%$ en 1997 al 72 \% en el 2001 (19). En el presente estudio la quimiorradiación, en concordancia con estas recomendaciones, fue el tratamiento elegido para la mayoría de las pacientes (41,4\%), y como primera alternativa para los estadios FIGO IB2, IIB y IIIB.

En 2002 se publicó un metaanálisis que mostraba un beneficio significativo para el grupo de quimioterapia neoadyuvante y cirugía comparado con la radioterapia sola, con una reducción del riesgo de muerte del 36\% y una mejora absoluta del $15 \%$ en la supervivencia a 5 años (20). En el presente estudio solo una de las 16 pacientes con estadio IB2 recibió quimioterapia neoadyuvante, siendo llevada posteriormente a histerectomía radical con linfadenectomía pélvica.

\section{Limitaciones del estudio}

Este estudio, por ser de tipo retrospectivo, es susceptible de tener sesgos de información; además, no se pudo determinar el impacto de los diferentes tipos de tratamiento en la sobrevida de las pacientes. En 27 de ellas no se pudo establecer el resultado en el tiempo. Esta pérdida en el seguimiento podría llevar a una sobrestimación de la sobrevida y el tiempo libre de enfermedad. Si se asume que las pacientes perdidas tuvieron un mal desenlace, la sobrevida podría estar cercana al $85 \%$.

\section{CONCLUSIONES}

Los resultados obtenidos en cuanto a complicaciones y sobrevida posterior al tratamiento fueron similares a lo reportado en la literatura. Se requieren más estudios con una mayor profundidad metodológica que caractericen el pronóstico de las pacientes con cáncer de cérvix en nuestra población. 


\section{REFERENCIAS}

1. Perkin DM, Bray F, Ferloy J, Pisani P. Eshmeting the world cancer burden globocan 2000. Int J Cancer. 2001:153-6.

2. Siegel R, Naishadham D, Jemal A. Cancer statistics, 2012. CA: A Cancer Journal for Clinicians. 2012; 62: 10-29. doi: 10.3322/caac. 20138

3. Departamento Administrativo Nacional de Estadística de Colombia (DANE). Estadísticas Vitales 2010 [visitado 2013 enero 13]. Disponible en http://www. dane.gov.co/index.php?option $=$ com_content\&view $=$ article\&id $=73 \&$ Itemid $=119$.

4. Ferlay J, Shin HR, Bray F, Forman D, Mathers C, Parkin DM. Globocan 2008 v2.0, Cancer Incidence and Mortality Worldwide: IARC CancerBase No. 10. Lyon, France: International Agency for Research on Cancer; 2010. [Visitado 2013 enero 13]. Disponible en: http://globocan.iarc.

5. Muñoz N, Franceschi S, Bosetti C, Moreno V, Herrero R, Smith JS et al. Role of parity and human papillomavirus in cervical cancer: the IARC multicentric case-control study. Lancet. 2002;359:1093-101.

6. Cogliano V, Grosse Y, Baan R, Straif K, Secretan B, El Ghissassi F. Carcinogenicity of combined oestrogenprogestagen contraceptives and menopausal treatment. Lancet Oncol. 2005;6:552-3.

7. De San José, Palacio V, Tafur LA, Vásquez S, Espitia VE, Vázquez F, et al. Prostitution, HIV, and cervical neoplasia: a survey in Spain and Colombia. Cancer Epidemiol Biomarkers Prev. 1993;2:531-5.

8. Thun MJ, Apicella LF, Henley SJ. Smoking vs other risk factors as the cause of smoking-attributable deaths: confounding in the courtroom. JAMA. 2000;284: 706-1.

9. Suprasert P, Srisomboon J, Kasamutsu T, Radical Histerectomy for stage IIB cervical cancer; a review. Int Gynecol Cancer. 2005;15:995-1001.

10. Marin F, Pleşca M, Bordea CI, Voinea SC, Burlãnescu I, Ichim E, et al Postoperative surgical complications of lymphadenohysterocolpectomy. J Med Life. 2014;7:60-6.
11. Wang X, Liu R, Ma B, Yang K, Tian J, Jiang L, et al. High dose rate versus low dose rate intracavity brachytherapy for locally advanced uterine cervix cancer. Cochrane Database of Systematic Reviews 2010, Issue 7. Art. No.: CD007563. doi: 10.1002/14651858.CD007563. pub2.

12. Zobec-Logar HB, Segedin B, Hudej R. Definitive radiotherapy for uterine cervix cancer: Long term results for patients treated in the period from 1998 till 2002 at the Institute of oncology Ljubljana. Radiot Oncol. 2013;47:280-8.

13. Green JA, Kirwan JJ, Tierney J, Vale CL, Symonds PR, Fresco LL, et al. Concomitant chemotherapy and radiation therapy for cancer of the uterine cervix (review). The Cochrane Library 2012. Issue 2012.

14. The International Collaboration of Epidemiological Studies of Cervical Cancer (2007). Comparison of risk factors for invasive squamous cell carcinoma and adenocarcinoma of the cervix: collaborative reanalysis of individual data on 8,097 women with squamous cell carcinoma and 1,374 women with adenocarcinoma from 12 epidemiological studies. Int J Cancer. 2006;120:885-91.

15. Parkin DM, Whelan SL, Ferlay J, Teppo L, Tomas DB, et al. Cancer incidence in five continents, vol. VIII. Lyon: IARC Press; 2002.

16. Lagasse LD, Creasman WT, Shingleton HM, Blessing JA. Results and complications of operative staging in cervical cancer: experience of the Gynecology Oncology Group. Gynecol Oncol. 1980;9:90-8.

17. Hockel M, Horn L-C, Fritsch H. Association between the management compartment of uterovaginal organogenesis and local tumor spread in stage IB - IIB cervical cancer: a prospective study. Lancet Oncol. 2005;6:751-6.

18. Chen Y, Xu H, Zhang Q, Li Y, Wang D, liang Z. A fertilypreserving option in early cervical carcinoma: laparoscopy-assisted vaginal radical trachelectomy and pelvic lynphadenectomy. Eur J Obstet Gynecol Reprod Biol. 2008;108:90-93. 
19. Trimble EL, Harlan LC, Gius D, Stevens J, Schawartz SM. Patterns of care for women with cervical canecr in the United States. Cancer. 2008;113:743-9.
20. Neoadjuvant Chemotherapy for Cervical Cancer Metaanalysis Collaboration. Neoadjuvant chemotherapy for locally advanced cervical cancer: a systematic review and metaanalysis of individual patient data from 21 randomized trials. Euro J Cancer. 2003;39:2470-86. 